\title{
Prevalencia de factores de riesgo cardiovascular en ingresantes universitarios
}

\author{
Prevalence of cardiovascular risk factors in \\ first year university students
}

\author{
Carlos A. Girotto, Marta N. Vacchino, Cynthia A. Spillmann y Jorge A. Soria \\ Universidad Nacional de Mar del Plata. Argentina (C.A.G.), Instituto Nacional de Epidemiología “Dr. \\ Juan H. Jara". Mar del Plata. Argentina. (M.N.V., C.A.S., J.A.S.)
}

\begin{abstract}
Resumen
Con el objetivo de conocer la prevalencia de factores de riesgo para enfermedades cardiovasculares (ECV) y su relación con antecedentes familiares de enfermedad, se estudiaron 3.357 ingresantes al 1er año de la Universidad de Mar del Plata, en el año 1991 que completaron su revisación médica obligatoria. La prevalencia de antecedentes familiares de hipercolesterolemia, hipertensión, diabetes mellitus, obesidad, enfermedad cardiovascular (ECV) fue: $27,5 \%, 42,1 \%, 26,9$ $\%, 27,2 \%$ y $42,1 \%$ respectivamente. El 80,5\% de los alumnos reportó al menos uno de los antecedentes familiares estudiados. La prevalencia de hipertensión (presión arterial sistólica $>140 \mathrm{mmHg}$ y/o presión arterial diastólica $\geq 90 \mathrm{mmHg}$ ) y de hipercolesterolemia ( $\geq 210 \mathrm{mg} / \mathrm{dl}$ ) en los participantes fue 7,0\% y $14,4 \%$. Se observó asociaciones de hipertensión con Indice de masa corporal (IMC), sexo masculino y edad. El 27,1\% manifestó su condición de fumador, no presentándose diferencias entre sexos. El hábito se correlacionó positivamente con la edad y se observaron distintas prevalencias según la carrera universitaria elegida. La presencia de colesterolemia aumentada se correlacionó con la edad, el IMC y los antecedentes familiares de obesidad (OR: 1,32 IC95\% $=1,06-$ $1,64)$ e hipercolesterolemia (OR:1,38 IC95\% = 1,10-1,69). Se detectó en un $3,7 \%$ anormalidades en el aparato cardiovascular. Se observó asociación con antecedentes de enfermedad cardíaca familiar. La prevalencia de factores de riesgo cardiovasculares hallados representan un toque de atención dada la juventud de la población considerada y muestran la necesidad de insistir en medidas educativas y de promoción de conductas preventivas.
\end{abstract}

Enfermedades cardiovasculares, epidemiología. Factores de riesgo. Estudiantes.

\section{Abstract}

The prevalence of cardiovascular risk factors and their relation to a self-reported family history of disease was examined in 3,357 first year university students of Mar del Plata University (Argentine). The prevalence of family disease was 
$27.5 \%$ for hypercholesterolemia, $42.1 \%$ for hypertension, $26.9 \%$ for diabetes mellitus, $27.2 \%$ for obesity and $42.1 \%$ for cardiovascular disease. The percentual of $80.7 \%$ of the population surveyed showed at least one of these diseases in their previous family history. The prevalence of hypertension (systolic blood pressure levels $\geq 140 \mathrm{mmHg}$ ) or/and diastolic blood pressure levels $\geq 90 \mathrm{mmHg}$ ) was $7.0 \%$. Hypertension was related to Body Mass Index (BMI), male sex and age. The percentual of $14.4 \%$ presented hypercholesterolemia $(\geq 210 \mathrm{mg} / \mathrm{dl})$, which was associated with age, BMI and family history of obesity and hypercholesterolemia. Nine hundred and eleven subjects (27.1\%) were smokers. Differences related to sex were not found. Smoking was positively related to age and the career they had chosen. The examination detected one hundred and twenty-three $(3.7 \%)$ students with cardiac problems. This was associated with a family history of cardiovascular disease. Preventive measures were suggested.

Cardiovascular diseases, epidemiology. Risk factors. Students.

\section{Resumo}

Com o objetivo de conhecer a prevalência de fatores de risco para doenças cárdiovaculares (ECV) e sua relação com antecedentes familiares de doença, estudaram-se 3.357 ingressantes ao primeiro ano da Universidade de Mar del Plata, no ano de 1991, que completaram seu exame médico obrigatório. A prevalência de antecedentes familiares de hipercolesterolemia, hipertensão, diabetes mellitus, obesidade, doenças cárdio-vasculares foi de 27,5\%, 42,1\%, $26,9 \%, 27,2 \%$ e $42,1 \%$, respectivamente. Oitenta por cento dos alunos reportaram, ao menos, um dos antecedentes familiares estudados. A prevalência de hipertensão (pressão arterial sistólica $>140 \mathrm{mmhg}$ e/ou pressão arterial diastólica $\geq 90 \mathrm{mmhg}$ ) e de hipercolesterolemia ( $\geq 210 \mathrm{mg} / \mathrm{dl}$ ), nos participantes, foi de $7,0 \%$ e 14,4\%. Observaram-se associações de hipertenasão com índice de massa corporal (IMC), sexo masculino e idade. A prevalência do hábito de fumar foi de $27,1 \%$, não apresentando diferenças entre sexos. O hábito correlacionou-se positivamente com idade e observaram-se diferentes prevalências segundo a carreira escohida. A presença de colesterolemia aumentada correlacionou-se com a idade. O IMC e os antecedentes familiares de obesidade $(\mathrm{OR}=1,32$ IC95\% $=1,06$ a 1,64) e hipercolesterolemia $(\mathrm{OR}=1,38$ IC95\%=1,10-1,69). Detectaram-se $3,7 \%$ anormalidades no aparelho cardiovascular. Observou-se associação com antecedentes de doença cardíaca familiar. A prevalência de fatores de risco-vasculares representa uma advertência, dada a juventude da população considerada, e mostra a necessidade de insistir em medidas educativas e de promoção de condutas preventivas.

Doenças cardiovasculares, epidemiologia. Fatores de risco. Estudantes.

\section{INTRODUCCIÓN}

Las enfermedades cardiovasculares (ECV) se encuentran en los primeros lugares como causa de muerte a nivel mundial. En el año 1992 ocuparon el 1er. lugar entre las causas de muerte en Argentina, representando el $30 \%$ de las muertes totales, con una tasa general de 790,7/10.000, manteniendo el primer lugar para el grupo de edad mayor de 50 años, segundo lugar para el grupo de 15-49 años y tercer lugar en el de 1-14 años.
En la actualidad, un individuo que viva en una sociedad postindustrial tiene una posibilidad sobre tres de estar afectado por una enfermedad cardiovascular y una sobre cuatro de morir a causa de ella ${ }^{8}$.

Argentina tal como otros paises en desarrollo, presenta un patrón de mortalidad que sugiere una transición epidemiológica desde una situación en que la mortalidad es dominada por las enfermedades infecciosas, a otro modelo de país en el que las enfermedades crónicas vinculadas a los factores de riesgo 
derivados de la conducta y las crónicas degenerativas como las cardiovasculares son las causas dominantes de muerte.

En el Partido de Gral Pueyrredón (en el que está situada la ciudad de Mar del Plata) ya en el año 1980 las enfermedades cardiovasculares representaron la primera causa de muerte y primera causa de años de vida potencialmente perdidos ${ }^{22}$.

Las enfermedades cardiovasculares tienen una etiología multifactorial. Los grandes estudios prospectivos clásicos (Framinghan, Tecumseh) ${ }^{7}$ y otros de tipo transversal y retrospectivos ${ }^{1,2,3,6,8,9,10,13-20}$ han permitido descubrir las asociaciones más importantes entre algunas variables y las ECV.

En primer lugar: el abuso del tabaco, la hipertensión arterial y la hipercolesterolemia.

En segundo lugar: la diabetes, la obesidad y la inactividad.

El tipo de comportamiento constituye probablemente la clave en la etiología de la enfermedad coronaria.

En su mayoría son factores exógenos (75\%). Los factores genéticos representan alrededor del $25 \%$ de la etiología.

La importancia de los factores hereditarios es mal conocida. Se cree que la herencia puede ser importante en la tensión arterial, la tolerancia a la glucosa, el aumento de ácido úrico y los triglicéridos plasmáticos, mientras que en el seno de las familias, los factores ambientales estarían relacionados con las lipoproteínas, el colesterol total y el hematocrito. Se han efectuado estudios en los que se observó relación entre la historia familiar de riesgo y enfermedad cardiovascular ${ }^{3}, 18$.

En la Argentina se ha estudiado la prevalencia de uno o más de estos factores de riesgo en grupos seleccionados, pero en general la comunicación de este tipo de información es escasa y los estudios no son comparables.

En investigaciones realizadas en Buenos Aires ${ }^{13} \mathrm{y}$ La Plata ${ }^{4}$ se encontró que la prevalencia de hipertensión arterial en adultos jóvenes estaba entre el 8 y $12 \%$. En estudios realizados en Universidades Norteamericanas se encontró una prevalencia de $12-18 \%$ de hipercolesterolemia en alumnos ingresantes al primer año ${ }^{5}$.

Ante la imposibilidad de establecer una prueba experimental mediante un ensayo controlado, la alternativa de estudio de esta problematica puede ser el control de un factor existente (colesterolemia, hipertensión) y el monitoreo de la disminución del riesgo consiguiente ante la patología cardiovascular.

Hasta ahora los programas de prevención han sido justificados por estudios experimentales de laboratorio, las encuestas y estudios de la variación concomitante de la enfermedad y de los factores de riesgo probables.
El control de la hipertensión arterial y del abuso de tabaco presenta buenas perspectivas en la prevención de la enfermedad. Se estima que el abandono del hábito de fumar podría controlar cerca del $30 \%$ de las muertes por ECV y salvar cerca del $24 \%$ de los años de vida potencial perdidos. La modificación de los comportamientos, alimentarios en particular, parece difícil de conseguir.

En cuanto al hábito de fumar información registrada en Chile y Brasil ${ }^{12}$ muestra que la prevalencia de fumadores fué mayor en alumnos de ciencias de la salud de $7^{\circ}$ año respecto a los de $1^{\circ}$ año. En otra investigación realizada en universitarios de la Universidad de Rosario (Argentina) ${ }^{15}$ se observó mayor prevalencia de fumadores en estudiantes de Medicina que en estudiantes de Ciencias Económicas. Esto presenta una situación paradojal, ya que aquéllos de quienes se esperaría un mayor énfasis en la prevención, de acuerdo a sus conocimientos sobre el tema salud, se exponen más.

En suma, la atención dispensada a estas enfermedades en los estudios epidemiológicos está plenamente justificada por la amplitud del problema.

Con el propósito de contribuir al conocimiento del tema y proveer información de base para futuros programas preventivos, se realizó un estudio descriptivo y de casos y controles para detectar factores de riesgo de ECV en ingresantes a la Universidad Nacional de Mar del Plata, que completaron su exámen clínico en el año 1991.

\section{Objetivos de la Investigación}

- Determinar la prevalencia de factores de riesgo para enfermedades cardiovasculares como hipertensión arterial, hipercolesterolemia, práctica deportiva, hábito de fumar, obesidad y de afecciones cardiovasculares en ingresantes a la Universidad en el año 1991.

- Determinar la prevalencia de antecedentes familiares de factores de riesgo.

- Detectar y analizar correlaciones entre presencia de factores de riesgo en el ingresante y entre éstos $\mathrm{y}$ antecedentes familiares.

- Estudiar posible correlación entre afección cardíaca actual y antecedentes familiares.

\section{MATERIAL Y METODO}

Se estudió una población de 3.357 estudiantes de ambos sexos ingresantes a la Universidad Nacional de Mar del Plata, en el año 1991.

Se realizó un exámen preventivo. Para tal fin se confeccionó una historia clínica de cada estudiante. En la 
anamnesis se registraron entre otros datos: sexo, edad, procedencia, antecedentes de enfermedades familiares, habito de fumar y actividad deportiva.

Se realizó además un exámen físico con medición de altura, peso, pulso, visión, presión arterial y un exámen clínico, del cuál el presente trabajo presenta solo los resultados de la observación del aparato cardiovascular, consignándose la presencia de soplos, ruidos anormales y transtornos del pulso.

Se extrajo a cada ingresante (previo ayuno de $12 \mathrm{hs)}$ una muestra de sangre para dosar colesterol total. Las extracciones se realizaron en el Servicio de Salud de la Universidad (en el caso de los ingresantes residentes en la ciudad) y en laboratorios particulares (demás casos).

Las muestras provenientes del Centro de Salud, se procesaron en el Laboratorio del Instituto Nacional de Epidemiología "Dr Juan H. Jara". En aquellas muestras procesadas que presentaron valores de colesterolemia $\geq$ $210 \mathrm{mg} / \mathrm{dl}$, se determinó la concentración de HDL Colesterol, Triglicéridos, relación Col. Total/HDL y concentración de LDL Colesterol mediante la ecuación de Friedenwaald.

Las determinaciones se realizaron utilizando equipos comerciales basados en métodos enzimáticos.

Se consideraron los siguientes valores normales:

- Colesterol total: hasta $210 \mathrm{mg} / \mathrm{dl}$.

- HDL Colesterol: Hombres: > 35 mg/dl;

- Mujeres: > $45 \mathrm{mg} / \mathrm{dl}$.

- Relación Colest.T/HDL Col.: Hombres:3.8-5.9; Mujeres: 3.1-4.6.

- LDL Col: riesgo normal: 150-190mg/dl.

- Triglicéridos: 10 a $150 \mathrm{mg} / \mathrm{dl}$.

La presión arterial se midió con el participante sentado, en el brazo derecho, con dos controles a intervalos de 15 min. Se tomó como valor definitivo el correspondiente a la 2 da toma.

Se consideró la clasificación propuesta por el Comité de Detección, Evaluación y Tratamiento de la Hipertensión de los EEUU²:

Presión arterial sistólica (PAS): normal: $\leq 140 \mathrm{mmHg}$; Hipertensión sistólica leve: 140-159 mmHg; Hipertensión sistólica: $\geq 160 \mathrm{~mm} \mathrm{Hg}$.

Presión arterial diastólica (PAD): normal: $\leq 85 \mathrm{mmHg}$; normal alta: 85-89 mmHg; hipertensión leve: 90-104 mmHg; hipertensión moderada: 105-114 mmHg; hipertensión severa : $\geq 115 \mathrm{mmHg}$.

\section{Definiciones Utilizadas en el Análisis de Datos}

Hipercolesterolemia: Presencia de concentración de colesterol total en sangre $\geq 210 \mathrm{mg} / \mathrm{dl}$.

Fumador: quién fuma en la actualidad, independientemente de la cantidad consumida.

Años de fumador: diferencia entre edad actual y edad de comienzo de la adicción.

Realización de deporte: quién realiza algún tipo de deporte, independientemente de si lo realiza diariamente o ocasionalmente.
Antecedentes familiares de enfermedades: antecedentes de diabetes mellitus, hipertensión, obesidad, hipercolesterolemia y enfermedades cardiovasculares padecidos por padres, abuelos o hermanos de sangre.

Indice de Masa Corporal (IMC): Cociente entre el peso y el cuadrado de la talla. Se considera aconsejable $\leq 24 \mathrm{~kg} / \mathrm{m} 2$. Hipertensión: Presión arterial sistólica $>140 \mathrm{mmHg}$ y/o Presión arterial diastólica $\geq 90 \mathrm{mmHg}$.

Alteración del aparato cardiovacular: Se consideró positiva cuando se observó la presencia de soplos funcionales o patológicos, ruidos anormales, pulsación acelerada u otra anomalía detectada mediante el exámen clínico.

En la transformación de las variables categóricas a numéricas la presencia de la variable se representó con la unidad y la ausencia con cero. El sexo masculino se representó con 1 y el femenino con cero.

\section{Análisis Estadístico}

Toda la información obtenida se procesó y analizó por computadora, utilizando programa estadistico Epi Info 5 para el estudio descriptivo, análisis de asociaciones de variables: Odd Ratio (O.R), $\chi 2$, coeficiente de correlación (r) y regresión lineal múltiple.

Después de la revisión del comportamiento de las variables estudiadas respecto a la presión arterial sistólica (PAS) y diastólica (PAD) se eligieron las más relevantes y se efectuó regresión lineal múltiple (técnica de análisis mutivariado que permite quitar a una variable la influencia de otras, previniendo de esta manera los efectos de confusión), considerando variables independientes el IMC, sexo, la edad y la PAS o PAD según el caso.

Lo mismo se realizó con IMC, considerandola dependiente, se la analizó respecto a antecedentes familiares de obesidad, edad, sexo, PAD y PAS.

En una segunda etapa se realizó regresión logística por pasos (forward) con programa BMDP (Biomedical Program), método marginal. Límites utilizados para entrar y remover las variables del modelo: valor "p" aplicado a los coeficientes, menor de 0,10 (entrar) y valor "p" mayor de 0,15 (remover). Se aplicó sobre 3 modelos.

En el primero se consideraron como casos (variable dependiente) a aquellos ingresantes que mostraron alteración en su aparato cardiovascular (presencia de soplos, ruidos anormales, pulsación acelerada u otra anomalía detectada mediante el exámen clínico) y controles a quienes no las presentaron.

Las variables independientes fueron edad, sexo, pulso, presión arterial diastólica antecedente familiares de ECV y diabetes mellitus.

En el segundo y tercero modelo, se desglosó la variable alteración del aparato cardiovascular. En el segundo modelo se consideró caso a aquéllos que presentaron soplos funcionales y patológicos. Se seleccionaron como variables independientes aquellas que aparecían como relevantes en el primer modelo, estos fueron: sexo, pulso, presión arterial diastólica, ruidos anormales, taquicardia y antecedentes familiares de ECV. 
En el tercero modelo se consideró caso a aquéllos que presentaron ruidos anormales. Las variables independientes: sexo, pulso, presión arterial diastólica, soplos, taquicardia y antecedentes familiares de ECV.

\section{RESULTADOS}

Se analizó la edad y sexo de los alumnos en el año 1991, que completaron su exámen preventivo de salud. Considerando los datos globales y discriminados por unidades académicas, el promedio general de la edad de los 3.357 estudiantes, fué de 20,93 años, con una mediana de 19 años, un modo de 18 años y una desviación standar de 5,89 años. El 85,9\% estuvieron comprendidos entre los 16 y los 24 años, el $11,4 \%$ entre los 25 y los 39 años y el $2,7 \%$ fueron mayores de 40 años (Tabla 1).

Correspondieron al sexo femenino el 57,8\% y al sexo masculino el $42,2 \%$. La edad promedio de las ingresantes de sexo femenino fué de 20,82 años y la de los ingresantes de sexo masculino fué de 21,08 años.

Con respecto al sexo se observaron variaciones considerables entre las diferentes Unidades Académicas (Fig. 1). Se encontró gran supremacía

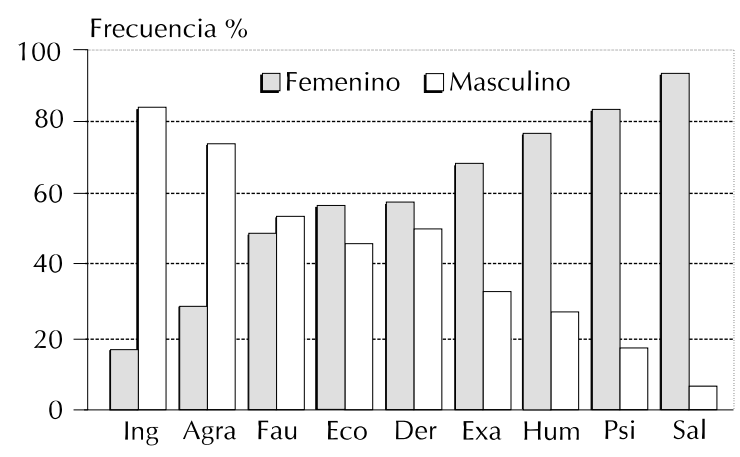

Figura 1 - Distribución s/facultad y sexo. Ingresantes a la Universidad Nacional de Mar del Plata. Ãno 1991. del sexo femenino en la Escuela de Ciencias de la Salud y del Comportamiento (93\%), Escuela Superior de Psicología $(81,6 \%)$ y Facultad de Humanidades. El sexo masculino predominó en la Facultad de Ciencias Agrarias (73\%) y en la de Ingeniería (83\%).

\section{Antecedentes Familiares Relacionados con Enfermedades Cardiovasculares}

El 80,5 \% de los ingresantes declaró uno o más antecedentes familiares: diabetes, obesidad, hipertensión, enf. cardiovasculares y/o colesterolemia aumentada.

Considerando los mismos en forma independiente: Diabetes 26,9\%; Obesidad 27,2\%; Hipertensión 42,1\%; ECV 42,1\%; Colesterol 27,5\%.

Los cuatro primeros antecedentes se asociaron positivamente con el antecedente de colesterolemia elevado $\left(\chi 2=41,48, \chi 2=46,38, \chi 2=28,32, \chi^{2}=73,72\right.$ respectivamente, en todos los casos resultaron altamente significativas $\mathrm{p}<0.0001)$. Los antecedentes de obesidad e hipertensión se asociaron positivamente con los antecedentes familiares de ECV $(\chi 2=28,68 \mathrm{p}$ $<0,0001$ y $\chi 2=50,31 \mathrm{p}<0,00001$, respectivamente).

\section{Actividades Deportivas}

El 48,1\% de los alumnos practicaba deportes. La actividad deportiva se mostró correlacionada positivamente al sexo masculino ( $\mathrm{r}=0,22$ IC95\%: $0,19$ a 0,25$)$ y negativamente al aumento de la edad $(r=-0,04$ IC95\%: - 0,01 a - 0,07).

Las Facultades que presentaron preponderancia de matrícula masculina y menor edad como Ciencias Agrarias e Ingeniería mostraron mayor proporción de deportistas $(74,6 \%$ y $51,9 \%)$ mientras que C. de la Salud y Psicología registraron menor actividad deportiva $(36,4 \%$ y $40,7 \%)$.

Tabla 1 - Distribución por edad y unidad académica. Ingresantes a la Universidad de Mar del Plata. Argentina. Año 1991.

\begin{tabular}{lcccc}
\hline Facultad & \multicolumn{2}{c}{ Cantidad de Alumnos } & \multicolumn{2}{c}{ Edad } \\
& $N^{\text {o }}$ & $\%$ & Promedio & DS \\
\hline Derecho (Der) & 1.175 & 35,0 & 21,89 & 7,28 \\
C. Económicas (Eco) & 709 & 21,2 & 19,22 & 2,92 \\
Psicología (Psi) & 359 & 10,7 & 22,11 & 6,55 \\
Arquitectura (Fau) & 277 & 8,3 & 20,05 & 2,66 \\
Ingeniería (Ing) & 259 & 7,7 & 19,13 & 2,07 \\
C. de la Salud (Sal) & 186 & 5,5 & 22,65 & 7,12 \\
C.E. y Naturales (Exa) & 169 & 5,0 & 18,88 & 2,26 \\
Humanidades (Hum) & 159 & 4,7 & 24,25 & 8,49 \\
C. Agrarias (Agra) & 63 & 1,9 & 18,90 & 1,10 \\
\hline Total & 3.357 & 100,0 & 20,93 & 5,89 \\
\hline
\end{tabular}

DS - Deviación standar 
Sin embargo no toda la variación pudo ser explicada por estas dos características; realizando un análisis multivariado, siguió observándose algo de variación asociada al tipo de disciplina elegida.

\section{Hábito de Fumar}

El 27,1\% (911) de los encuestados manifestó su condición de fumador, el hábito se relacionó con la mayor edad y presentó distintos valores de prevalencia en las distintas Facultaderes (Derecho: 34,0\%, Psicología: 33,4\%, Ciencias de la Salud: $29,0 \%$, Arquitectura: 25,6\%, Ciencias Económicas: 21,6\%, Humanidades: 19,5\%, Ingeniería: 17,1\%, C. Exactas: $14,9 \%$ y C. Agrarias: $11,1 \%$ ) (Tabla 2).

No existió diferencia estadísticamente significativa entre proporción de hombres y mujeres fumadores. Los hombres en promedio comienzan a fumar algo antes que las mujeres.

El número de cigarrillos diarios fumados también se incrementó con la edad ( $\mathrm{r}=0,21$ IC95\%: 0,15-0,27) $\mathrm{y}$ con los años de fumador ( $\mathrm{r}=0,29$ IC95\%: 0,23-0,35).

\section{Exámen de Presión Arterial}

El 96,5\% de los 3.357 examinados presentó valores de presión arterial sistólica iguales o menores a 140 $\mathrm{mmHg}$; el 2,5\% valores de $140-160 \mathrm{mmHg}$; y el $1 \%$ presentó cifras iguales o superiores a $160 \mathrm{mmHg}$.

El 96,2\% de los alumnos presentó valores de tensión arterial diastólica menores a $90 \mathrm{mmHg}$; el 3,7\% valores de 90-104 mmHg (hipertensión leve) y menos del $0,1 \%$ registró valores superiores a 104 $\mathrm{mmHg}$ (hipertensión moderada y severa).

El 7,0\% de los ingresantes presentaron hipertensión, considerandose esta como: presencia de presión sistólica $>140 \mathrm{mmHg}$ y/o presión distólica $\geq 90 \mathrm{mmHg}$.

En la Figura 2 se representan las distribuciones observadas de presión sistólica y diastólica.

Se observó que la PAS se asoció a la PAD, al sexo masculino, al incremento de IMC (en este orden de importancia) (Tabla 3).

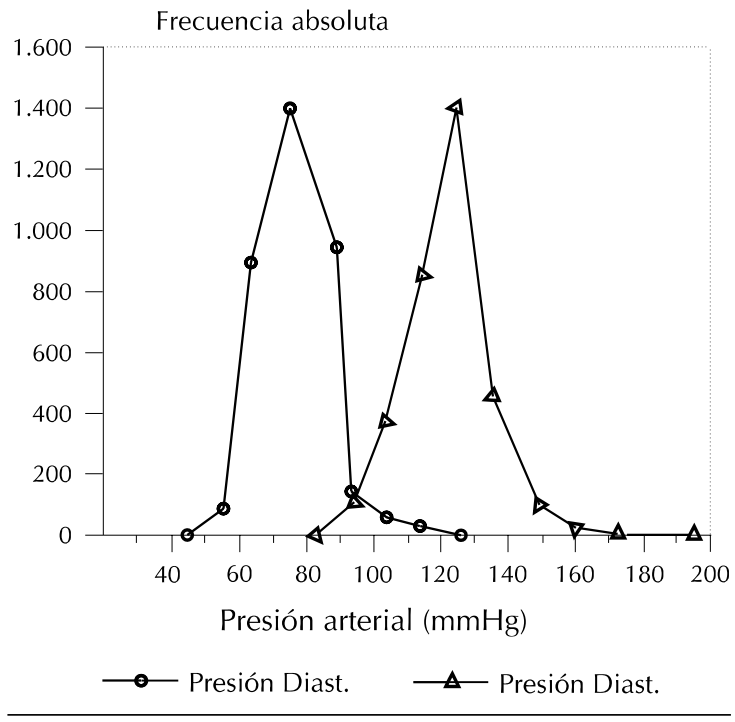

Figura 2 - Presión arterial sistólica y diastólica. Ingresantes a la Universidad Nacional de Mar del Plata - 1991.

La PAD se asoció significativamente al aumento de la PAS, al IMC, a la edad y al sexo masculino.

\section{Determinación de Lípidos}

El 14,4\% de las muestras de sangre del total de 3.357 alumnos ingresantes, mostraron valores de colesterol total en sangre iguales o mayores a $210 \mathrm{mg} / \mathrm{dl}$.

La concentración promedio de colesterol en sangre fue $178,6 \mathrm{mg} / \mathrm{dl}$, D.S $=31,5 \mathrm{mg} / \mathrm{dl}$, observándose un valor mínimo de $86,7 \mathrm{mg} / \mathrm{dl}$ y máximo de $400 \mathrm{mg} / \mathrm{dl}$.

La presencia en los alumnos de valores de colesterol en sangre iguales o mayores a $210 \mathrm{mg} / \mathrm{dl} \mathrm{se}$ asoció con la declaración de antecedentes familiares de obesidad e hipercolesterolemia.

Considerando casos a aquellos ingresantes con colesterol elevado y como controles a los demás, la presencia de antecedentes de colesterolemia familiar incrementó en un $32 \%$ el riesgo de presentar a su vez hipercolesterolemia.

Tabla 2 - Hábito de fumar en ingresantes a la Universidad Nacional de Mar del Plata - 1991.

\begin{tabular}{|c|c|c|c|c|}
\hline Variables & Mínimo & Máximo & Promedio & DS \\
\hline \multicolumn{5}{|l|}{ Edad de comienzo (años) } \\
\hline Mujer & 10 & 45 & 16,7 & 2,7 \\
\hline Hombre & 11 & 42 & 16,5 & 2,5 \\
\hline \multicolumn{5}{|l|}{ Años de fumador } \\
\hline Mujer & $<1$ & 32 & 5,4 & 6,1 \\
\hline Hombre & $<1$ & 48 & 5,9 & 6,2 \\
\hline \multicolumn{5}{|c|}{$\mathrm{N}^{\circ}$ de cigarrillos fumados por día } \\
\hline Mujer & 1 & 60 & 9,4 & 7,5 \\
\hline Hombre & 1 & 72 & 11,9 & 8,0 \\
\hline
\end{tabular}

DS - Deviación standar 
Tabla 3 - Regresión lineal múltiple de presión arterial sistólica (PAS) y diastólica (PAD) en función de los factores de riesgo más significativos.

\begin{tabular}{lccc}
\hline Variable & Coefic. $\beta$ & IC $95 \%$ & Test F parcial \\
\hline PAD & 0,657 & $0,618-0,696$ & $1099,2^{*}$ \\
Sexo & 4,363 & $3,696-5,03$ & $164,3^{*}$ \\
IMC & 0,537 & $0,426-0,647$ & $90,7^{*}$ \\
Edad & 0,026 & $-0,029-0,082$ & 0,9 \\
\hline Variable dependiente: PAS & $\mathrm{n}=3.344$ & $\mathrm{R} 2=36,7 \%$ & $1099,2^{*}$ \\
\hline PAS & 0,377 & $0,354-0,399$ & $28,5^{*}$ \\
IMC & 0,300 & $0,145-0,314$ & $27,6^{*}$ \\
Edad & 0,112 & $0,070-0,154$ & $4,4^{*}$ \\
Sexo & 0,550 & $0,034-1,068$ & \\
\hline Variable dependiente: PAD & $\mathrm{n}=3.344$ & $\mathrm{R} 2=32,5 \%$ & \\
\hline
\end{tabular}

* Estadísticamente significativo.

IMC - Indice de Masa Corporal.

IC - Intervalo de confianza.

Tabla 4 - HDL Colesterol, LDL Colesterol, relación HDL/Colesterol y triglicéridos en ingresantes con Colesterol Total > de $210 \mathrm{mg} / \mathrm{dl} . \mathrm{N}=383$

\begin{tabular}{rcccc}
\hline \multicolumn{1}{l}{ Variables } & Mínimo & Máximo & Promedio & DS \\
\hline HDL Colesterol (mg/dl) & & & & \\
Mujer & 33,3 & 133,3 & 68,3 & 15,9 \\
Hombre & 29,4 & 135,5 & 59,5 & 15,4 \\
Mujer & & & & \\
Relación Col/HDL & 1,7 & 8,1 & 3,6 & 0,9 \\
Hombre & 2,0 & 7,7 & 4,1 & 1,0 \\
TDL Colesterol (mg/dl) & 80,5 & 312,0 & 148,6 & 25,2 \\
Triglicéridos (mg/dl) & 33,6 & 371,7 & 97,2 & 41,6 \\
\hline
\end{tabular}

DS - Deviación standar

Asimismo las personas con antecedentes familiares de obesidad presentaron un riesgo $38 \%$ superior

Antec. Colesterolemia

Antec. Obesidad

"Odds Radio"
1,32
1,38

También se observó correlación simple significativa entre aumento de concentración de colesterol y edad ( $\mathrm{r}=0,21$ IC95\% = 0,18 a 0,25) e Indice de Masa Corporal (IMC) $(r=0,14$ IC95\% $=0,10$ a 0,25$)$.

Esto indica que a mayor edad y mayor IMC se incrementa el valor del Colesterol. Realizando un análisis de regresión múltiple de conc. de colesterol en función de las variables citadas sigue siendo significativa la asociación.

El HDL colesterol se determinó a aquellos sueros de alumnos cuya extracción se realizó en el Servicio Universitario de Salud y presentaron cifras de colesterol total iguales o mayores a $210 \mathrm{mg} / \mathrm{dl}$. Sobre un total de de presentar hipercolesterolemia que aquellos que no presentaron estos antecedentes.

$\begin{array}{crc}\text { IC } 95 \% & \text { Chi2 } & p \\ 1,06-1,64 & 6,83 & 0,009 \\ 1,10-1,69 & 8,76 & 0,003\end{array}$

383 muestra analizadas, el promedio de HDL colesterol fué de $64,7 \mathrm{mg} / \mathrm{dl}$, con un valor mínimo de $29,4 \mathrm{mg} / \mathrm{dl}$ y un valor máximo de $135,1 \mathrm{mg} / \mathrm{dl}$ (Tabla 4).

Se considera valor de riesgo en hombres por debajo de $35 \mathrm{mg} / \mathrm{dl}$ y en mujeres inferior a $45 \mathrm{mg} / \mathrm{dl}$.

Se efectuó el cálculo de la relación colesterol total/ HDL colesterol para establecer los grupos de riesgo aterogénicos, registrándose un total de 30 casos en el sexo femenino con un valor mayor de 4.6.

En el sexo masculino se encontraron 6 casos con relación mayor a 5,9. Tanto los 30 casos de sexo femenino y los 6 casos de sexo masculino fueron considerados con alto riesgo aterogénico. 


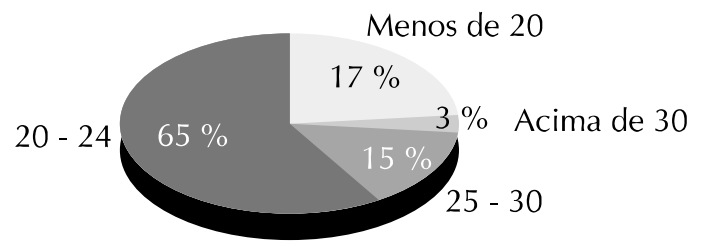

Indice Masa Corporal (IMC)

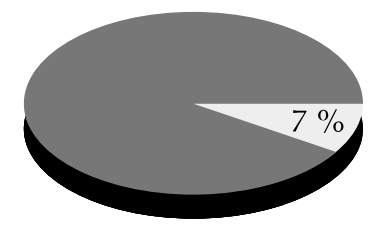

Hipertensión

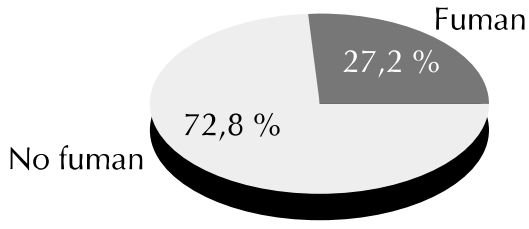

Hábito de fumar
Menos de $210 \mathrm{mg} / \mathrm{dl}$

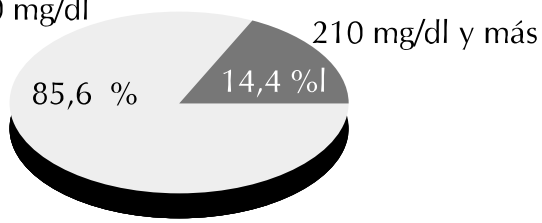

Colesterolemia

Figura 3 - Prevalencia de factores de riesgo. Ingresantes a la Universidade Nacional de Mar del Plata. Año 1991.

Se observó correlación negativa entre los valores de HDL colesterol y triglicéridos.

Cuando se relacionó estos datos con los antecententes familiares de hipercolesterolemia se encontró que la presencia de dichos antecedentes se asociaba a conc. menores de HDL Col y a conc. mayores de Triglicéridos.

\section{Resumen de Prevalencias de Factores de Riesgo Relevantes (Fig. 3)}

El 18,0\% mostró IMC superior al valor $24 \mathrm{~kg} /$ $\mathrm{m} 2$, aconsejado en la bibliografía. El valor promedio de IMC fue: $22,7 \mathrm{~kg} / \mathrm{m} 2$ y DS: $3,1 \mathrm{~kg} / \mathrm{m} 2$.

A través de regresión lineal múltiple se encontró que el IMC (considerado como variable dependiente) se asoció fuertemente a los antecedentes familiares de obesidad, al aumento de la edad, PAS, sexo masculino del ingresante y PAD (en este orden de importancia). (Test parcial $\mathrm{F}$ de los coeficientes: 153,$64 ; 100,29 ; 86,49 ; 47,73 ; 28,52$ respectivamente, todos ellos estadísticamente significativos).

\section{Prevalencia de Factores de Riesgo en Ingresantes Menores de 25 Años (Fig. 4)}

Se calculó las prevalencias de fumadores, hipercolesterolemia y hipertensión en ingresantes menores de 25 años $(n=2.886)$ y se calculó la prevalencia conjunta de dos de estos factores:

Prevalencia de fumadores

Prevalencia de hipercolesterolemia

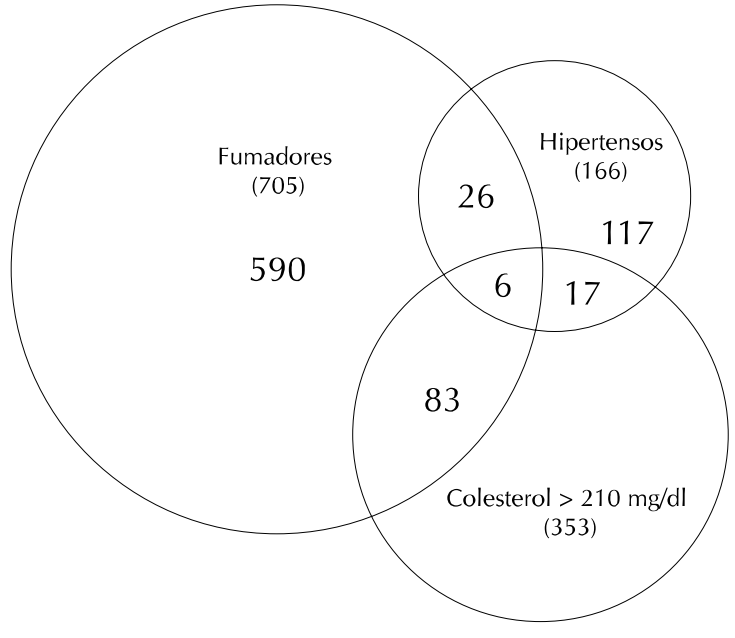

Figura 4 - Prevalencia de factores de riesgo en menores de 25 años.

Prevalencia fumadores y colesterolemia $>210 \mathrm{mg} / \mathrm{dl}$ $2,9 \%$ Prevalencia fumadores e hipertensión $\quad 0,9 \%$ Prevalencia hipertensión y colesterolemia $>210 \mathrm{mg} / \mathrm{d} \mathrm{l}$ $0,5 \%$

\section{Aparato Cardiovascular}

El 3,7\% (123) de los ingresantes mostró algún tipo de alteración cardíaca como soplos sistólicos funcionales y patológicos, ruidos anormales y/o trastornos en el pulso perisférico (radial).

El modelo $\mathrm{n}^{\circ} 1$ muestra que las mujeres presentaron un riesgo de alteraciones cardíacas $99 \%$ superior a los 
Tabla 5 - Regresión logística por pasos. Modelo número $1 *$.

\begin{tabular}{lccc}
\hline Variables seleccionadas & Coef. $\beta$ & O.R & IC \\
\hline Sexo (femenino) & 0,345 & 1,99 & $1,32-2,99$ \\
Presión diastólica & 0,036 & 1,07 & $1,03-1,12$ \\
Pulso & 0,018 & 1,04 & $1,01-1,08$ \\
Antec. de ECV & 0,195 & 1,47 & $1,03-2,12$ \\
\hline
\end{tabular}

$\mathrm{N}=3.314 \quad$ Casos $=123$

* Variable dependiente: presencia de alteraciones cardiovasculares. Variables independientes: edad, sexo, pulso, presión arterial diastólica, antecedentes familiares de enf. cardiovascular (antec. ECV) y de diabetes mellitus.

OR - "Odds Ratio"

IC - Intervalo de confianza

ECV - Enfermedades cardiovasculares

Tabla 6 - Regresión logística por pasos. Modelo número 2*.

\begin{tabular}{lccc}
\hline Variables seleccionadas & Coef. $\beta$ & O.R & IC $95 \%$ \\
\hline Sexo femenino & 0,551 & 3,01 & $1,84-4,91$ \\
Presión diastólica & 0,050 & 1,10 & $1,05-1,17$ \\
Ruidos anormales & 1,441 & 17,84 & $5,19-61,36$ \\
\hline
\end{tabular}

$\mathrm{N}=3.314 \quad$ Casos $=97$

* Variable dependiente: presencia de soplos funcionales y patológicos. Variables independientes: sexo, pulso, presión arterial diastólica, ruidos anormales,

taquicardia y antecedentes familiares de enfermedad cardiovascular.

Tabla 7 - Regresión logística por pasos. Modelo número 3*.

\begin{tabular}{|c|c|c|c|}
\hline Variables seleccionadas & Coef. ß & O.R & IC $95 \%$ \\
\hline Antec. de ECV & 1,028 & 7,81 & $1,72-35,49$ \\
\hline Soplos & 1,323 & 14,09 & $4,21-47,15$ \\
\hline
\end{tabular}

$\mathrm{N}=3.314 \quad$ Casos $=13$

* Variable dependiente: presencia de ruidos anormales. Variables independientes: sexo, pulso, presión arterial diastólica, soplos, taquicardia y antecedentes familiares de enfermedad cardiovascular (antec. de ECV).

hombres, aquellos con antecedentes familiares de ECV un "Odds ratio" (OR) 47\% superior y por cada unidad de incremento de PAD el riesgo se incrementó en un 7\%. El pulso resultó no significativo (Tabla 5).

Cuando se utilizó el modelo nำ2, la presencia de soplos se correlacionó al sexo, auscultación de ruidos anormales y presión arterial diastólica (Tabla 6).

El riesgo de soplos en mujeres fue 3 veces mayor, en aquellos que presentaron ruidos anormales 17 veces superior y por cada unidad de incremento de PAD se incrementó en un $10 \%$.

En el modelo $\mathrm{n}^{\circ} 3$, se observó que en los ingresantes con antec. de ECV, el riesgo (OR) de ruidos anormales fué 7,8 veces el riesgo de aquellos sin antecedentes. Como era de esperar la auscultación de ruidos anormales se asocia fuertemente a la presencia de soplos (Tabla 7).

\section{DISCUSIÓN}

En este estudio se observa alta prevalencia de antecedentes familiares de factores de riesgo.

En el estudio Cardia (EEUU) llevado a cabo en población de ambos sexos comprendida entre 18-30 años ${ }^{3}$ se encontró una prevalencia de enfermedad parenteral de hipertensión, obesidad y diabetes de $44,2 \%, 47,1 \%$ y $10,7 \%$ respectivamente. A pesar de que nuestras referencias familiares fueron más amplias, ya que además de padres incluímos abuelos y hermanos de sangre, las prevalencias de antecedentes de hipertensión y obesidad son menores $(42,1 \%$, $27,2 \%)$, no así la de diabetes que resultó como era de esperar más alta $(26,9 \%)$.

En nuestro caso, al igual que en estudio citado la historia familiar de obesidad se relacionó con la presencia de colesterolemia aumentada y con incremento de $\mathrm{IMC}^{3}$; los antecedentes familiares de colesterol elevado se asociaron con el incremento de colesterol en el ingresante y en aquellos alumnos que presentaron colesterolemia con el incremento de triglicéridos y la disminución de HDL Colesterol.

La historia de ECV familiar se asoció con alteraciones del aparato cardiocirculatorio.

Los soplos sistólicos funcionales y patológicos se asociaron al sexo femenino, el aumento de presión arterial diastólica y presencia de ruidos anormales.

Hubiera sido importante consignar solamente los soplos considerados patológicos, pero el exámen fue 
realizado por médicos generales que hicieron el primer diagnóstico del caso y posteriormente efectuaron la derivación al especialista, no reciclándose posteriormente la información en forma homogénea. A pesar del sesgo que esta situación introduce, resulta interesante la observación de que la mayoría de los alumnos que presentaron a la auscultación ruidos cardíacos anormales, declararon antecedentes familiares de enfermedad cardiovascular $(\mathrm{OR}=7,81$ IC $95 \%=1,72-35,49)$.

Otro sesgo a considerar en el estudio es que el conocimiento de enfermedades familiares por parte del alumno posiblemente sea limitado.

Sin embargo el efecto que provocaría esta situación tendería a subestimar el verdadero riesgo, ya que posiblemente el no conocimiento de enfermedad se traduciría en la declaración de una historia negativa de la misma, por lo que la asociación resultaría subestimada.

El hecho de que estos antecedentes familiares de enfermedad no han sido validados por médicos constituirían otra fuente de error, sin embargo existen estudios $^{6}$ que han detectado buena concordancia $(78 \%)$ entre historia familiar declarada y validación médica de los registros.

A pesar de ser una población eminentemente joven las prevalencias de colesterol elevado, hipertensión, y IMC $>24 \mathrm{~kg} / \mathrm{m}^{2}(14,4 \%, 7,0 \%, 18,0 \%$ respectivamente) son para considerar, más aún cuando estas no se reducen demasiado al limitar nuestra población a los menores de 25 años (12,2\%, $5,7 \%, 15,5 \%$ respectivamente).

Además se observan asociaciones positivas entre la hipertensión arterial, el IMC, edad y sexo masculino; y entre colesterol elevado, IMC y el incremento de edad.

Semejantes asociaciones han sido descriptas por otros investigadores ${ }^{1,9,17}$. Casi la tercera parte de los estudiantes fuman. La tasa de fumadores es comparable a la hallada en estudiantes de medicina en Rosario, Argentina (29\%) $)^{15}$ y en Brasil $(28 \%)^{21}$ y menor a la de estudiantes de la salud en Chile ${ }^{11}$. Al igual que en el estudio de Rosario, se observaron diferencias en la prevalencia del hábito en distintas disciplinas. En dicho estudio los estudiantes de Medicina mostraron mayor prevalencia que los de Ciencias Económicas. En nuestro caso las tasas mayores se observaron en carreras de tipo humanístico como Derecho y Psicología y la menores en Ciencias Exáctas y Agrarias. La Universidad de Mar del Plata no posee Facultad de Medicina, por lo que sólo se pudo comparar la prevalencia en Ciencias Económicas, resultando muy parecidas (22\%-Rosario vs 21,6\%-Mar del Plata).

No hubo diferencias entre sexos. La edad de inicio del hábito resultó semejante en hombres y mujeres (16,5 vs 16,7 años). Esto estaría de acuerdo con la tendencia de aumento de frecuencia de tabaquismo en mujeres, observada en Argentina y otros paises sudamericanos en los últimos decenios.

El consumo diario de cigarrillos se incrementó con los años de fumador, o sea que a inicio más temprano mayor cantidad de cigarrillos diarios fumados.

Esta característica, que se reitera en otros trabajos, está señalando la necesidad de concentrar los esfuerzos contra este hábito en el nivel escolar primario y la adolescencia. El patrón de las enfermedades asociadas al tabaquismo, en Argentina, es similar al de los países con población muy expuesta al tabaquismo. Se calcula que anualmente se producen de 38.000 a 49.000 muertes atribuídas al uso de tabaco ${ }^{12}$.

Estos resultado obligan a una reflexión en cuanto a preguntarnos por un lado que papel cumple el paso por la Universidad respecto a la evolución de estos factores de riesgo y por otro lado que tipo de estrategias conducentes a la reducción de los mismos podrían desarrollarse ya que se acumulan en la literatura artículos que relatan el efecto benéfico a largo plazo de diversas medidas preventivas principalmenmente de abordaje multifactorial ${ }^{2,5,17,18,19}$.

\section{REFERENCIAS BIBLIOGRÁFICAS}

1. BAUMGARTNER, R. et al. Fatness and fat patterns associations with plasma lipids and blood pressures in adults, 18 to 57 years of age. Am. J. Epidemiol., 126: 61428, 1987.

2. BJARTWEIT, K. et al. Cardiovascular screenings in Norwegian counties: trends in risk patter during the period 1985-90 among persons aged 40-42 in 4 counties. TidsskrNor-Laegeforen., 117(17): 2076-6, 1991.
3. BURKE, G.L. et al. Relation of risk factor levels in young adulthood to parental history of disease; the CARDIA Study. Circulation., 84: 1176-87, 1991.

4. ECHEVERRÍA, R.F. et al. Prevalencia de hipertensión arterial en La Plata. Medicina (Buenos Aires), 48: 22-8, 1988.

5. FAIGEL, H.C. Screening college students for hypercholesterolemia. J. Am. Coll. Health, 40:272-5, 1992. 
6. FORDE, O.H. \& THELLE, D.S. Themso Heart Study: risk factors for coronary heart disease related to ocurrence of myocardial infarction un first degree relatives. Am. J. Epidemiol., 105: 192-9, 1977.

7. DAWBER, T.R. The framingham study. Cambridge, Harvard University Press, 1980.

8. JENICEK MILOS, C.R. Epidemiología: principiostécnicas- aplicaciones. Editorial Masson-Salvat, 1993.

9. LANAS, F. et al. Prevalencia de factores de riesgo cardiovascular en la Ciudad de Temuco. Rev. Méd. Chile, 119: 247-51, 1991.

10. LOLIO, C. et al. Hipertensão arterial e possíveis fatores de risco. Rev. Saúde Pública, 27: 357-62, 1993.

11. MEDINA, E.L. \& KAEMPFFER, A.M. Tabaquismo y salud en Chile. Bol. Ofic. Sanit. Panamer, 100: 112-20, 1991.

12. ORGANIZACIÓN PANAMERICANA DE LA SALUD. Tabaco o salud: situación en las Américas. Washington, 1992. (OPS-Pub. Científ. nº 536).

13. ORGANIZACIÓN PANAMERICANA DE LA SALUD. $L a$ hipertensión arterial como problema de salud comunitario. Washington, 1990. (Manuales Paltex).

14. POLETTO, L. et al. Blood lipid associations in year-old men. Rev. Saúde Pública, 26: 316-20, 1992.
15. POLETTO, L. et al. Prevalence of smoking in young people and their parents. Important associations with education and occupation. Rev. Saúde Pública, 25: 388-93, 1991.

16. PYÖRÄLA. K. Coronary heart disease: difference in the occurrence between populations; relationship to serum colesterol levels. In: Scott M, et al, ed. The role of cholesterol in atherosclerosis. Hanley and Belfus Inc., 1987.

17. ROCHA NOGUEIRA, et al. Colesterol e otros factores de risco cardiovascular nos servidores da UFRJ: prevalencia e influencia de variaveis sociais. Arq. Bras. Cardiol., 55: 227 32,1990

18. SCIENTIFIC STEERING COMMITTEE ON BEHALF OF THE SIMON BROOME REGISTER GROUP. Risk of fatal coronary heart disease in familiar hypercholesterolemia. BMJ., 303 (6807), 1991.

19. SEIM, A. et al. Prevention of cardiovascular diseases in Rissa. Tidsskr-Nor-largeforen, 111(17): 2078-80, 1991.

20. SIGFUSSON, N. et al. Decline in risk factor levels. BMJ., 302 (6789): 1371-5, 1991.

21. SZEGO, T. et al. Levantamento epidemiológico sobre o vicio de fumar entre estudantes de medicina no Estado de São Paulo. Rev. Ass. Méd. Bras., 31: 13-6, 1985.

22. VACCHINO, M. \& SACHUMEYER, E. Mortalidad en Pto de Gral Pueyrredón: años 1970-80. Publicación Instituto Nacional de Epidemiología "Dr Juan H. Jara”, 1990. 